This work has been submitted for publication. Copyright may be transferred without further notice, and this version may no longer be accessible.

\title{
Estimating a Mean Ocean State from Hydrography and Sea-Surface Height Data with a Non-linear Inverse Section Model: Twin Experiments with a Synthetic Data Set
}

\author{
Martin Losch ${ }^{1,2}$, René Redler ${ }^{3,4}$, and Jens Schröter ${ }^{1}$
}

\begin{abstract}
The recovery of the oceanic flow field from in situ data is one of the oldest problems of modern oceanography. In this study, a stationary, non-linear inverse model is used to estimate a mean geostrophic flow field from hydrographic data along a hydrographic section. The model is augmented to improve these estimates with measurements of the absolute sea-surface height by satellite altimetry. Measurements of the absolute sea-surface height include estimates of an equipotential surface, the geoid. Compared to oceanographic measurements, the geoid is known only to low accuracy and spatial resolution, which restricts the use of sea-surface height data to applications of large scale phenomena of the circulation. Dedicated satellite missions that are designed for high precision, high resolution geoid models are planned and/or in preparation. Our study, which relies on twin experiments, assesses the important contribution of improved geoid models to estimating the mean flow field along a hydrographic section. When the sea-surface height data are weighted according to the error estimates of the future highly accurate geoid models GRACE (Gravity Recovery And Climate Experiment) and GOCE (Gravity field and steady-state Ocean Circulation Explorer) integrated fluxes of mass and temperature can be determined with an accuracy that is improved over the case with no sea-surface height data by up to $55 \%$. With the error estimates of the currently best geoid model EGM96, the reduction of the estimated flux errors does not exceed $18 \%$.
\end{abstract}

\section{Introduction}

Estimating the mean ocean circulation is one of the major tasks of physical oceanography. Wunsch [1978] and many later studies [e.g. Rintoul, 1991; Macdonald, 1998; Sloyan and Rintoul, 2001] provide one approach

\footnotetext{
${ }^{1}$ Alfred-Wegener-Institut für Polar- und Meeresforschung, Bremerhaven, Germany

${ }^{2}$ Institut für Meereskunde, Kiel, Germany

${ }^{3}$ current affiliation: Department of Earth, Atmospheric, and Planetary Sciences, Massachusetts Institute of Technology, Cambridge

${ }^{4}$ current affiliation: C\&C Research Laboratories NEC Europe Ltd., Sankt Augustin, Germany
}

to determine the mean steady-state circulation from hydrographic section data. The method of these studies uses in-situ measurements along hydrographic sections and employs thermal wind and inverse techniques to obtain an estimate of the large scale, low frequency flow through those sections. In this approach, the vertical shear of velocity is determined directly from the in-situ measurements and geostrophy, that is the balance of Coriolis force and horizontal pressure gradient force. Consequently possible measurement errors or systematic errors that arise when one tries to estimate a mean state from quasi-synoptic data are interpreted as implicit model errors. All errors are formally assigned to the unknown additive reference velocity and to mass 
imbalances [Ganachaud and Wunsch, 2000].

The natural extension to that approach is to treat temperature, salinity, and passive tracers as control parameters that are to be fitted to the in-situ measurements. It is then possible to allow certain deviations (or errors) of these variables from the data a priori. One could then estimate mean tracer fields from the in-situ data and from physical assumptions, to which prior errors can be attributed. On the basis of such mean tracer fields, the estimate of the mean velocity field, derived transport estimates, and their estimated errors are more reliable. For not only do they include the uncertainty of the reference velocity but also the errors of the mean tracer field estimates. A model that includes this extension has been used by Nechaev and Yaremchuk [1995], Yaremchuk et al. [1998], and Yaremchuk et al. [2001] to estimate a mean state of the ocean along a single section from the hydrographic data of multiple repeat cruises.

To our knowledge the model of Nechaev and Yaremchuk [1995] is the first and so far only inverse section model that estimates new tracer fields along with the velocity field. A few studies employ three dimensional models in which density is a control parameter [e.g., LeGrand, 2001] to estimate a mean state from hydrographic data. Zhang and Hogg [1996] and de las Heras and Schlitzer [1999] assume in their inverse models that the thermal wind balance is not exact, which introduces an explicit description of the model error. However, none of these studies are devoted to analyzing hydrographic data along an isolated section. The same holds true for the numerous assimilation studies, which use general circulation models.

In this paper, we investigate the ability of a geostrophic model similar to that of Yaremchuk et al. [2001] to estimate a mean state along a single hydrographic section with data of only one hydrographic cruise. Clearly, hydrographic data from a single cruise will not be sufficient to estimate a mean state. Instead, we make use of additional types of data for which time series are available. Two data types immediately come to mind: long-term current measurements from moorings and satellite based altimetric measurements of the sea-surface height. Of these two types, sea-surface height measurements provide potentially the best resolution and coverage for all ocean regions. Sea-surface height also proves to be useful as in the geostrophic context its slope is directly proportional to the geostrophic surface velocity. Ganachaud et al. [1997] explored the combination of TOPEX/Poseidon altimetry and hydrographic data with a box inverse model. In their model, hydrographic measurements and geostrophy fix the vertical velocity shear, as described above. Our approach includes the use of sea-surface height as well but we employ an inverse model that allows deviations of temperature, salinity, and tracer fields from the measurements. Thus, our model can handle uncertainties (e.g., associated with the steady-state assumption) explicitly by allowing model data misfits a priori.

With the advent of altimetric data of extraordinary accuracy and precision from the TOPEX/Poseidon or ERS2 mission, measurements of sea-surface height data are at hand. To date, this information has mostly been used in studies of the time-dependent phenomena in the ocean [e.g. tides or ocean variability, see Wunsch and Stammer, 1998, for a review] where the absolute sea-surface height is not needed. For the use of satellite altimetry data in the steady-state ocean problem, one has to reference the altimetric measurement to a marine geoid in order to determine the mean dynamic sea-surface height. Unfortunately, the uncertainties associated with the presently available marine geoids exceed those of the altimetry by an order of magnitude that renders the mean sea-surface height very noisy [Ganachaud et al., 1997].

While the present geoid accuracies limit the use of satellite altimetry on the determination of the mean ocean circulation, two dedicated satellite missions, which will determine an accurate geoid, are now funded or proposed: Gravity Recovery and Climate Experiment (GRACE) [Tapley, 1997] and Gravity field and steady-state Ocean Circulation Explorer (GOCE) [Battrick, 1999]. Given that in the near future we will have an accurate marine geoid, we can now explore the impact of accurate sea-surface height data on the analysis of hydrographic sections. Obviously, there is no accurate sea-surface height data now, but estimates of the errors of the future marine geoids are very well available. Therefore, we choose to use - along with these error estimates of the marine geoid - a synthetic data set that provides a complete and consistent ensemble of hydrography, current measurement, and sea-surface height data. This choice enables us to assess the impact of the new geoid models without the actual data.

Inverse models are often tested using synthetic data and dynamics. These studies range from testing the applicability of inverse models in general [Thacker, 1989] to "validation" of particular inverse models [McIntosh and Rintoul, 1997]. In fact, Nechaev and Yaremchuk [1995] tested their model - the first published version of the model presented here - with data from the FRAM atlas [Webb et al., 1991]. Zhang and Hogg [1996] used 
data from a GCM to test their inverse model. Most studies report, that the inverse models are able to reproduce the synthetic data within estimated errors, although there are a few studies where the inverse models fail their tests, as reported by Zhang and Hogg [1996].

From the synthetic data set, which is produced by an ocean general circulation model (OGCM) of the North Atlantic Ocean, a mean ocean state can be estimated. In our case, it will be characterized by fluxes of mass and temperature through parts of a hydrographic section along approximately $24^{\circ} \mathrm{N}$. In a twin experiment, the inverse model estimates these fluxes from a synoptic snapshot of hydrographic data, representing a single "cruise", and from selected velocity "measurements" and eventually sea-surface height data averaged over one model year. The inverse model makes these estimates by fitting the model to the data, allowing certain model and data errors a priori. For sea-surface height data, these errors include the error estimates of the different marine geoids available: that of the stateof-the-art geoid model EGM96, and those of GRACE and GOCE. Because the resolution of the inverse model is higher than that of the sea-surface height data for all of the geoid models, particular care must be taken so that the model is only fitted to long scales determined by the sea-surface height data.

Model and approach are very similar to the second part of Schröter et al. [2001]. Here, however, we divide the section plane into five parts. This division allows us to assess the vertical and horizontal impact of high precision sea-surface height data. While Schröter et al. are primarily interested in their impact on the error reduction of net mass and temperature fluxes through the section, we emphasize the impact on the fluxes themselves and their resemblance to the reference state. It is not clear whether or not a synoptic section in conjunction with time-averaged sea-surface height data can be used successfully to estimate a mean state. Furthermore we take into account errors due to the model assumptions: for example, the deviation of a mean velocity, as estimated from hydrography and geostrophy, from the "true" mean, which includes all of the physics of the OGCM. In this context, we analyze the posterior error covariances of the inverse model to aid the interpretation of the results. Although the original data used in all experiments are exactly the same, we anticipate different solutions for different prior data error estimates because the model is underdetermined and non-linear. This makes regularization necessary, which will not allow to same closeness of fit to data for different data errors.
Section 2 briefly describes the synthetic data set used in this study and the OGCM that produced it. Section 3 outlines the basic properties of the inverse model and the design of the prior error covariances. Special attention is paid to the error covariances of the sea-surface height data because the treatment of those errors is different to, for example, Ganachaud et al. [1997]. For a more thorough description of the model, the reader is referred to the appendix. In Section 4 the twin experiments and their results are described. The summary and conclusion are given in Section 5 .

\section{FLAME Model Description: "Data"}

The data for the twin experiments of Section 4 were obtained by integration of the $(1 / 3)^{\circ}$ North Atlantic Model of the the FLAME group [Redler et al., $1998]^{1}$. The code of this ocean general circulation model (OGCM) is derived from MOM2 [Pacanowski, 1995] and extended for use on massive parallel computer architectures. In this text, we will call the model the FLAME-model (as opposed to the inverse model described in Section 3) for brevity.

The model area extends from $18^{\circ} \mathrm{S}$ to $70^{\circ} \mathrm{N}$ in the Atlantic Ocean. The grid has a resolution of $(1 / 3)^{\circ}$ meridionally and $(1 / 3)^{\circ} \cos \phi$ zonally ( $\phi$ denotes latitude). In the vertical, the separation between the 45 grid levels increases from $10 \mathrm{~m}$ near the surface to $225 \mathrm{~m}$ below $2250 \mathrm{~m}$ depth. At the surface, the model is driven by monthly mean values of ECMWF re-analysis data of wind stress and surface fluxes of heat and fresh water [Barnier et al., 1995]. The model is spun up from initial conditions based on $(1 / 4)^{\circ}$ horizontal resolution annual mean potential temperature and salinity fields [Boyer and Levitus, 1997] for a period of 10 years. After spin-up, snapshots of temperature, salinity, and the three-dimensional velocity fields are stored in intervals of three days. At the same time, three-day means of the sea-surface height are stored.

For our purposes the output of the FLAME-model is considered as "reality". This is very convenient because in contrast to the real ocean this "model ocean" is very well sampled. A subset of the model ocean is treated as measurements from which the inverse model estimates velocities. We can then compare the estimate of the flow field by the inverse model to the flow field of the FLAME "model reality". Here we will not discuss the degree of resemblance of the FLAME-model output to the real Atlantic Ocean; to our belief, the FLAME-

\footnotetext{
${ }^{1}$ http://www.ifm.uni-kiel.de/fb/fb1/tm/research/FLAME/index.html
} 


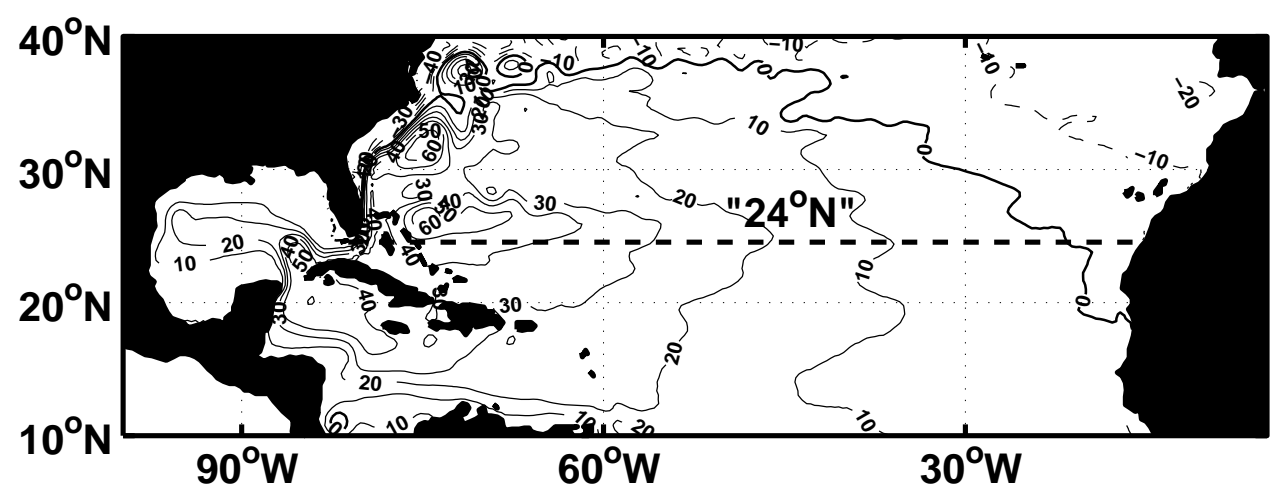

Figure 1. Part of the model area of the FLAME $1 / 3^{\circ}$ North Atlantic Model and location of the "section" at $24^{\circ} 30^{\prime} \mathrm{N}$. Contours show the mean sea-surface height for the eleventh model year; contour interval is $20 \mathrm{~cm}$.

model can simulate the Atlantic Ocean fairly well. However, one has to be aware that the physics incorporated in the FLAME-model and the inverse model are different so that one cannot expect perfect agreement between the two results. At the same time, this difference in physics is not the same as the difference between the physics of the inverse model and the physics of the real ocean [Zhang and Hogg, 1996]. On the other hand, here we will use only integrated transports for evaluating the performance of the inverse model that are less sensitive to the specific model configurations than, for example, velocity values at individual grid points.

From the data set we extract a synoptic snapshot at $24^{\circ} 30^{\prime} \mathrm{N}$, approximately at stations identical to the WOCE section A5 (see Fig. 1). This provides us with hydrography and sea-surface height data that are consistent within the dynamics of the FLAME model. A reference mean state is constructed from a one-year mean of the eleventh model year. At the time of the snapshot, January 3, of the eleventh model year, the instantaneous net mass flux through the section is $\phi_{V}=-0.4 \mathrm{~Sv}\left(1 \mathrm{~Sv}=10^{6} \mathrm{~m}^{3} / \mathrm{s}\right)$, the one-year mean $\overline{\phi_{V}}=-0.2 \pm 0.3 \mathrm{~Sv}$, the instantaneous temperature transport relative to $0^{\circ} \mathrm{C}$ is $\phi_{\theta}=0.73 \mathrm{PW}$ $\left(1 \mathrm{PW}=10^{15} \mathrm{~J} / \mathrm{s}\right)$ and its one-year mean value $\overline{\phi_{\theta}}=$ $0.74 \pm 0.13 \mathrm{PW}$. The uncertainties are the standard deviations of the respective one-year means. The temperature flux values of the FLAME-model are smaller than recent temperature flux estimates across the real section A5 of 1.1 to 1.3 PW by Roemmich and Wunsch [1985], Bryden [1993], Macdonald and Wunsch [1996], and Ganachaud and Wunsch [2000]. However, the differences between the FLAME-model and the real ocean do not affect our study, because - as stated above we regard the FLAME simulation as "reality". For a discussion of the heat transport in a similar model of the North Atlantic, the reader is referred to Willebrand et al. [2001] and Böning et al. [2001]. The net mass flux through a zonal section of the FLAME is zero by construction. However, the values obtained here are calculated after subsampling and interpolating the velocity onto the grid of the inverse model (see Section 3). Therefore, one can expect small sampling errors. For the net mass flux this error is well within one standard deviation.

Figure 2 shows the temporal average of the spatial power spectral density of the one-year sea-surface height record and the same for the vertically integrated mass and temperature transports, the quantities of interest in this study. Large portions of the signal have a wavelength of 200 to $500 \mathrm{~km}$. In particular, this wavelength band accounts for $58 \%$ of the mass flux variance and $46 \%$ of the temperature flux variance along the section. Although current estimates of the sea-surface height do not resolve this spectral band properly we can still use these data because the sea-surface height spectrum is "much more red" so that the 200-500 km band describes only $5 \%$ of its variance. The sea-surface height estimates are limited by the coarse resolution of the stateof-the art geoid model EGM96. Future geoid models will have a resolution high enough to resolve the spectral range down to $200 \mathrm{~km}$ wavelength (see Section 4). Below $200 \mathrm{~km}$ the spectra drop sharply. As Böning and Budich [1992] noted, this is approximately the length scale where diffusion starts to dominate in a $(1 / 3)^{\circ}$ model with biharmonic parameterization of horizontal mixing.

In this paper, we will use integrated transports for evaluating the performance of the inverse model. The 


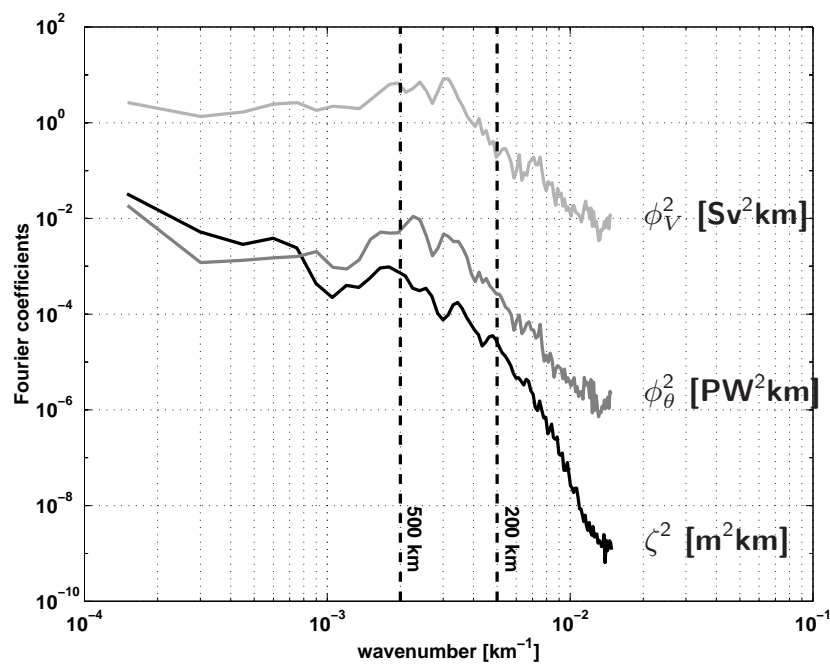

Figure 2. One-year record: Power spectral density of sea-surface height, mass transport $\phi_{V}$, and temperature transport $\phi_{\theta}$ at $24^{\circ} 30^{\prime} \mathrm{N}$ of the FLAME $1 / 3^{\circ}$ North Atlantic Model.

hydrographic section is divided into five regions to resolve the major currents that pass through it. Fig. 3 shows these regions along with their mass flux values of the FLAME-model for the Florida Current (I), the Antilles Current (II), the deep western boundary current (IV), the return flow (III), and the deep sea (V).

Flux values calculated from the winter snapshot differ substantially from annual mean fluxes due to weaker density gradients in winter that lead to weaker deep western boundary currents. In this sense the choice of the winter snapshot turns recovering the mean circulation into a hard test for the inverse model.

\section{Inverse Section Model}

\subsection{Description of the model}

The model used in this investigation is a geostrophic inverse model based on the approach described in Nechaev and Yaremchuk [1995] and Yaremchuk et al. [1998]. Density is calculated from an estimate of temperature and salinity with the non-linear equation of state for sea water [Fofonoff and Millard, 1983]. The thermal wind equations determine the corresponding vertical profile of horizontal velocity except for an unknown constant. This constant, commonly called reference velocity, is constrained by a local advective conservation equation for potential temperature and salinity and the planetary vorticity equation (see Appendix A1).
The equations are discretized on a finite difference grid that "envelops" the hydrographic section (see Fig. 4): there is one grid plane on each side of the section consisting of tracer grid points. These are the positions of the (independent) model parameters temperature and salinity, from which density is calculated. Four of these grid points on each plane make up the eight vertices of a grid box in the center of which there is a horizontal velocity grid point. Vertical velocity grid points lie on the upper and lower faces of this grid box. On the western end of the section (the Florida Strait) the spacing of these boxes coincides with the grid size of the OGCM; east of $78^{\circ} \mathrm{W}$ it increases to $1^{\circ}$. The width of the boxes in the direction normal to the section is the average of their along-section size. In the vertical, the vertices of the boxes are also defined by the uneven GCM grid. From this grid the inverse model's estimates of temperature, salinity, velocity, and sea-surface height are mapped to the data locations between the grid planes.

Following the standard "assimilation philosophy", we consider all data as a kind of spatio-temporal sample of a random ensemble of the ocean states. By treating the large-scale steady state of the model ocean as an average over this ensemble, it is possible to find an approximation to this mean under the assumption that the prior probability distribution is Gaussian. In addition, a number of conservation laws and dynamical relationships must be satisfied [Yaremchuk et al., 1998]. These constraints comprise the model described above. Furthermore, an integral kinematic constraint imposes a net mass transport across the section.

Some of these constraints - namely the advective conservation equation, the vorticity balance and the transport constraint - are imposed in a weak form; that is they are to be satisfied only within certain error bounds defined by a priori statistical assumptions. The error bounds reflect the model errors and the uncertainty of the a priori transport estimate, both of which can be treated explicitly by our inverse scheme. On the other hand, since we are interested in the large scale, low frequency component of the circulation, geostrophy is assumed to be an accurate approximation. The error of the density equation of Fofonoff and Millard [1983] is smaller than $0.03 \mathrm{~kg} / \mathrm{m}^{3}$. Therefore, both density equation and geostrophy are satisfied exactly.

After specifying the probability distribution and the dynamical constraints, we invert the data by seeking the most probable state of the North Atlantic on our model grid under the assumption that the dynamical constraints are satisfied. The numerical technique for 


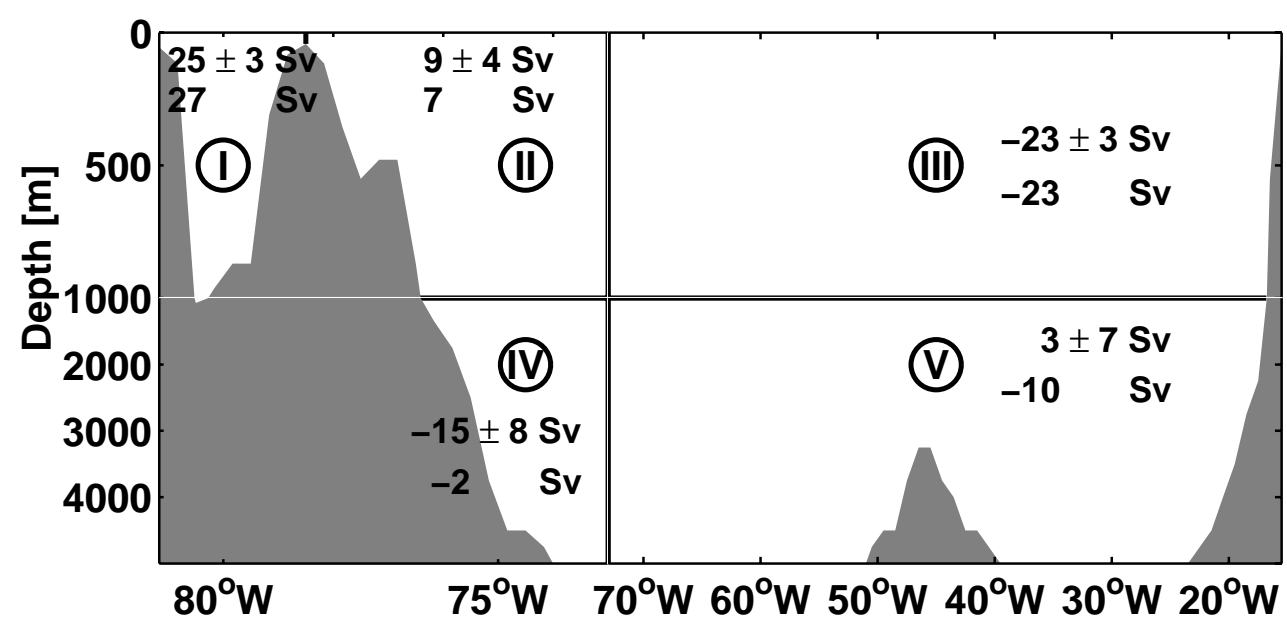

Figure 3. Section along $24^{\circ} \mathrm{N}$ through the FLAME model with division into five regions. Note the stretched coordinate system that emphasizes the regions above $1000 \mathrm{~m}$ and west of $72^{\circ} \mathrm{W}$. The upper numbers are the oneyear mean mass fluxes through the regions with standard deviation; the lower numbers are instantaneous values of the same fluxes at the time of the snapshot.

that procedure is well established [e.g., Le Dimet and Talagrand, 1986]. The prior probability density function is proportional to $\exp (-\mathcal{J})$ where the argument $\mathcal{J}$ has the form of the following quadratic cost function (for a more detailed description of the cost function see the appendix):

$$
\begin{aligned}
\mathcal{J}= & \underbrace{\mathcal{J}_{C}+\mathcal{J}_{\mathbf{u}}+\mathcal{J}_{\zeta}}_{\text {data terms }} \\
& +\underbrace{\mathcal{J}_{\boldsymbol{\tau}}+\mathcal{J}_{-H}+\mathcal{J}_{q}+\mathcal{J}_{\phi_{V}}}_{\text {weak constraints }} \\
& +\underbrace{\mathcal{J}_{\mathbf{u}_{\text {ref }}}+\mathcal{J}_{\partial_{y} C}+\mathcal{J}_{\hat{S} C}+\mathcal{J}_{\hat{S} q}+\mathcal{J}_{\hat{S} \mathbf{u}}}_{\text {regularization }}
\end{aligned}
$$

The first three terms in $\mathcal{J}$ attract the solution to the data. The subscripts $C, \mathbf{u}$, and $\zeta$ denote hydrographic parameters (temperature and salinity), horizontal velocities and sea-surface height, respectively. The model-data misfits are weighted with weighting matrices, which are the inverse covariance matrices accounting for the prior statistical structure of the hydrographic properties $\left(W_{m n}\right)$, the horizontal velocity field $\left(W_{\mathbf{u}}\right)$, and the sea-surface height $\left(W_{\zeta}\right)$. In the case of seasurface height, this matrix contains among other errors the error structure of the geoid to which real data would have been referenced (see Section 3.2.3).

The next four terms are labeled as weak constraints. They are included to reduce the imbalance of the vorticity equation via the boundary conditions for vertical velocity at the surface $\left(\mathcal{J}_{\boldsymbol{\tau}}\right)$ and at the sea floor $\left(\mathcal{J}_{-H}\right)$, the terms neglected in the advective tracer conservation $\left(\mathcal{J}_{q}\right)$ and the deviation of the model from the a priori estimate of the net mass transport $\left(\mathcal{J}_{\phi_{V}}\right)$. As these terms are a measure of the model error, they tend to reduce the model error.

Because the number of unknowns is large compared to the available information - twice as many model parameters $T$ and $S$ than measurements plus the unknown reference velocities and boundary conditions for vertical velocities-, additional assumptions about the unknown mean state have been made in order to regularize the under-determined problem. In particular, the model should not deviate too much from a priori estimates of the reference velocity $\left(\mathcal{J}_{\mathbf{u}_{\text {ref }}}\right)$ and the temperature and salinity gradients across the section $\left(\mathcal{J}_{\partial_{y} C}\right)$. In this paper, these estimates can conveniently be taken from the reference data set as produced by the OGCM. When analyzing real hydrographic sections, the temperature and salinity gradients across the section have to be estimated from a climatological atlas and a first guess of the reference velocities can be obtained by choosing a levelof-no-motion. Although we know the "true" values for these quantities in our scenario, our choice of weights for the corresponding cost function terms reflects the uncertainties one normally associates with them (see Section 3.2).

The remaining terms of the cost function are aimed at diminishing variances of the grid-scale components 


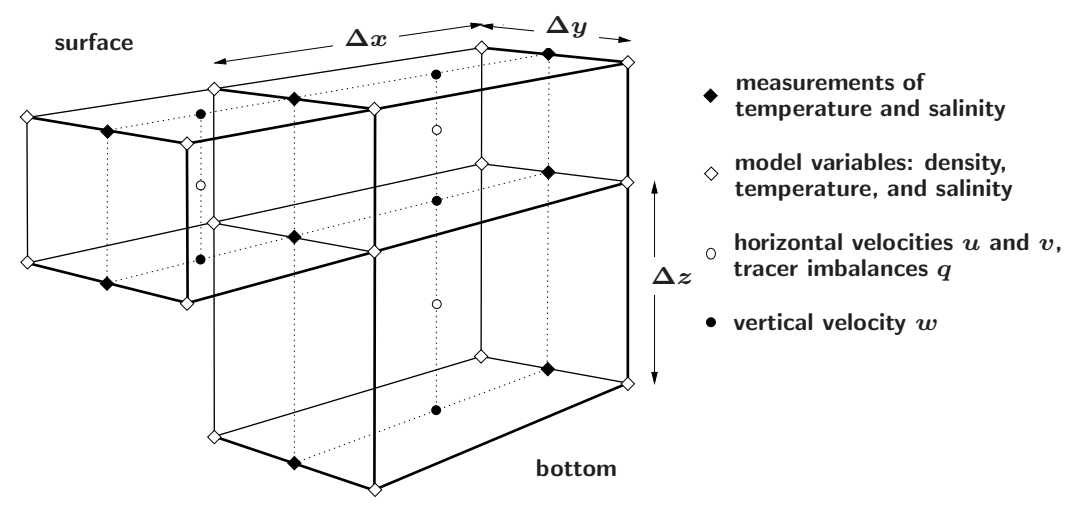

Figure 4. Sketch of the model grid. The solid lines connect the positions of the (independent) model parameters temperature and salinity. The dotted lines lie in the plane where the in situ data were collected.

of $C, q$, and $\mathbf{u}$ (smoothness of the fields). They are represented by applying the differential operator $\hat{S}_{\psi}:=$ $\partial_{x x}+\nu_{\psi}(z) \partial_{z z}$, where $\nu_{\psi}$ depends on the field $\psi$.

All weak constraints and regularization terms are weighted with diagonal weighting matrices. This constitutes the implicit assumption that a priori all arguments in the last nine quadratic terms are statistically independent and have zero means.

To find the maximum of the probability distribution we have to minimize the cost function $\mathcal{J}$. This optimization problem is solved iteratively using the adjoint method. The adjoint code necessary for this method was generated by the Tangent-linear Adjoint Model Compiler [TAMC, Giering, 1999].

\subsection{Prior Covariances}

This section discusses the design of the covariance matrices and particularly their inverses- $W_{m n}, W_{\mathbf{u}}$, $W_{\zeta}, W_{\boldsymbol{\tau}}, W_{-H}, W_{q}, W_{\phi_{V}}, W_{\mathbf{u}_{r e f}}, W_{\partial_{y} C}, W_{\hat{S} C}, W_{\hat{S} q}$, and $W_{\hat{S} \mathbf{u}}$ - to be used as weighting functions. These quantities define "physically acceptable" deviations of the model variables from their steady state geostrophically balanced counterparts. The weights $W$ should be inversely proportional either to the measurement error covariances $\left(W_{m n}, W_{\mathbf{u}}\right.$, and $\left.W_{\zeta}\right)$ or to the squared amplitudes of the fields that are not represented by the model equations (all others). We assume that all fields are statistically homogeneous in time and, with the exception of $W_{m n}$ and $W_{\zeta}$, also in space.

Most of the prior inverse covariances have been estimated in the same way as in Yaremchuk et al. [2001]. We therefore limit our discussion to those weights for which the calculation differs from that of Yaremchuk et al. [2001]. As almost all of the covariances are diag- onal, so are their inverses.

3.2.1. Tracer Weights The tracer weights $W_{m, n}$ are constructed in a way similar to that described by Yaremchuk et al. [2001]: first we construct covariance functions $C_{m, n}\left(z, z^{\prime}\right)$ that are local to their respective stations. They include vertical cross-correlations and tracer-tracer correlations because the vertical spacing of the grid resolves the scales of mesoscale eddies, which are treated as noise by the assimilation scheme. Including these correlations allows the model to change the depth of an isopycnal without changing the T-S relation on the isopycnal [Yaremchuk et al., 2001]. The local covariance functions are assumed to vary slowly along the section in order to account for east-west variations in hydrographic fields. Therefore, the covariances are smoothed by a Gaussian-bell-shaped running average over the along section coordinate of the section. Furthermore, an estimate of "ocean noise" according to Bindoff and McDougall [1994] has been added to the diagonal. Inverting the covariance matrix yields the weights to be used in the cost function.

The gradients of temperature and salinity normal to the section are one-year mean values of the FLAMEmodel data serving as a substitute for climatological data based on observations. The a priori errors are estimated from the horizontal variance per layer. The inverses of these errors are down-weighted by a factor of the order of 1000 that is proportional to the local grid box volume, so that the gradients have a smaller weight in the more variable small scale western boundary regions. In total, deviations from background values of the tracer gradients are only weakly penalized.

3.2.2. Velocity and Flux Constraints The Florida Current is very well monitored. To reflect the availability of velocity data in the Florida Strait, nine 
velocity data from the reference data set - three each at the three levels $37 \mathrm{~m}, 146 \mathrm{~m}$, and $748 \mathrm{~m}$-were used as contraints on the Florida Current. These velocity data represent mean value "measurements" from long-record current moorings; therefore, they are assigned a small error estimate of $1 \mathrm{~cm} / \mathrm{s}$. In many areas of the ocean, velocity measurements from moorings are not available and would have to be replaced by other data, if available (e.g. Acoustic Doppler Current Profiler data).

The net mass flux has been chosen to $0 \pm 1 \mathrm{~Sv} a$ priori $\left(W_{\phi_{V}}=1 \mathrm{~Sv}^{-2}\right)$ to account for the assumption of mass conservation in the North Atlantic. Although the FLAME model does not have any significant source of transport uncertainty across a zonal section, we use the relatively large prior uncertainty of $1 \mathrm{~Sv}$. By that we account for sampling errors because of the coarse resolution of the section and for "real" transport uncertainties that would be introduced if we dealt with data from the real ocean. These include uncertainties of the inflow through the arctic, difference between evaporation and precipitation, etc.. The temperature flux is not constrained.

The velocity at the deepest grid point between two stations is taken as the reference velocity $\mathbf{u}_{r e f}$. Its first guess (and background) value is taken from the FLAME-model at the same time as the hydrography. The temporal dispersion of its modulo $\int_{t_{0}}^{t_{1}}\left|\mathbf{u}_{r e f}\right|^{2} d t /\left(t_{1}-t_{0}\right)$ of the one-year time series serves as a priori error estimate. With this choice the a priori error for reference velocities ranges from $1-2 \mathrm{~cm} / \mathrm{s}$ in the deep basins to $10-25 \mathrm{~cm} / \mathrm{s}$ in the region of the western boundary currents.

3.2.3. Sea-Surface Height Weights The weighting matrix for the sea-surface height data term $\mathcal{J}_{\zeta}$ requires some additional care. Because dynamic sea-surface height (sometimes also called sea-surface topography) is the difference of the surface height $h$ over a reference ellipsoid and the geoid height $N$, its covariance $C_{\zeta}$ is the sum of the error covariances of the measurement of $h$ by altimetry and that of the geoid model, which provides the geoid undulation $N$. Unfortunately, $h$ and $N$ are available in different resolutions and representations. Therefore, their error covariances describe different scales. In addition to that, the inverse model's estimate of the sea-surface height has still another resolution. Of these three data types, the geoid model, which is given as coefficients of an expansion into spherical harmonic functions, is generally the one with the poorest resolution. Also, its large omission error, represented by high degree spherical harmonics, calls for a procedure that suppresses high degrees and short spatial scales.

We have chosen a filter that was proposed by Jekeli [1981] and recently used by Wahr et al. [1998] to investigate the geoid's time variability on long spatial scales. The kernel of this filter

$$
\begin{aligned}
K(\psi) & =\frac{b}{2 \pi} \frac{e^{-b(1-\cos \psi)}}{1-e^{-2 b}}, \\
b & =\frac{\ln (2)}{(1-\cos (r / R))},
\end{aligned}
$$

is nearly Gaussian - for large spherical distances $\psi$ it drops more slowly than the Gaussian bell curve - and has good attenuation properties for high degrees in the spectral domain of spherical harmonics. It is depicted in Fig. 5 for an averaging radius of $r=286 \mathrm{~km}$ that, by a rule of thumb, corresponds to a maximum spherical harmonic degree of $L=\pi R / r \approx 70 . R$ is the radius of the earth.

We proceed as follows: the geoid coefficient error covariance $\left\langle\delta c_{i} \delta c_{k}\right\rangle$ is transformed to a (nondiagonal) representation in spherical coordinates $x=(\lambda, \phi)$ :

$$
C_{N}\left(x, x^{\prime}\right)=\sum_{i, k}^{L} Y_{i}(x)\left\langle\delta c_{i} \delta c_{k}\right\rangle Y_{k}\left(x^{\prime}\right),
$$

where $Y_{k}(x)$ are the fully normalized spherical harmonic functions. The sum of the geoid error covariance $C_{N}$ and the altimetric error covariance $C_{h}$, which is assumed to be diagonal, yields an unsmoothed estimate of the sea-surface height error covariance $C_{\zeta}$. The Gaussian filter kernel (2) removes all scales shorter than the averaging radius $r$ from both "data" and the error covariance by

$$
\begin{aligned}
\overline{\zeta(x)} & =\int K(x, y) \zeta(y) d y \\
\overline{C_{N}\left(x, x^{\prime}\right)} & =\iint K(x, y) C_{N}\left(y, y^{\prime}\right) K\left(x^{\prime}, y^{\prime}\right) d y d y^{\prime} .
\end{aligned}
$$

The smoothed estimate $\bar{\zeta}$ along with its error covariance $\overline{C_{N}}$ is then restricted to the section coordinates.

The sea-surface height error covariance is generally ill-conditioned on the grid of the inverse model. Therefore, we construct the weighting matrix as a pseudoinverse of the covariance matrix by eigenvalue decomposition. Only those eigenvalues which are significantly different from zero are kept in building the weighting matrix. As the covariance matrix does not contain any small spatial scales, the resulting weighting matrix does not, either. This way, short scales effectively have zero weight and only the long scale components of the 
space domain

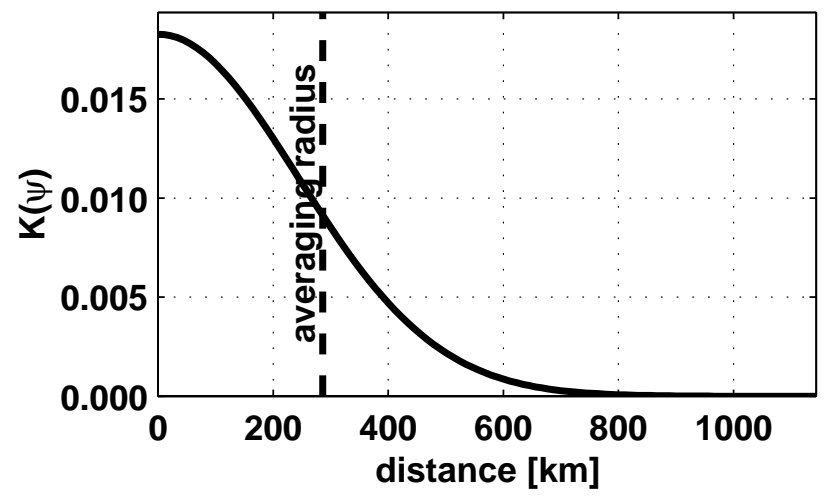

spectral domain

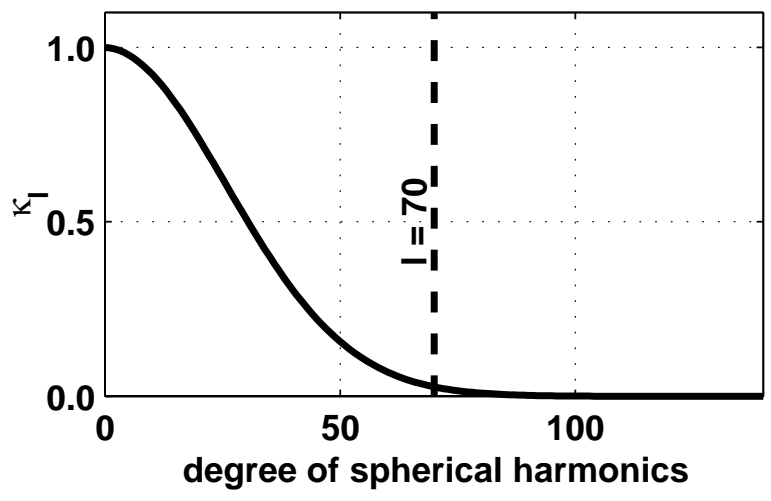

Figure 5. Left hand side: Quasi-Gaussian filter kernel $K(\psi)$, where $\psi$ is the spherical distance, for an averaging radius of $286 \mathrm{~km}$ [Jekeli, 1981]. This averaging radius corresponds to a geoid model resolution of degree $l=70$. Right hand side: Spectral expansion of the filter into spherical harmonic functions.

model's estimate are fitted to the long scale components of the sea-surface height data.

Geoid error estimates of three different geoid models were available. The first one is that of the state-of-theart geoid model EGM96 [Lemoine et al., 1997] ${ }^{2}$. The geoid model's coefficients are available up to degree 360 ; however, its error estimate is only complete to degree and order 70 . Therefore, we chose an averaging radius of $r=\pi R / 70 \approx 286 \mathrm{~km}$ for this geoid model.

The other two geoid models do not exist yet. They will be available when the dedicated gravity satellite missions GRACE [Tapley, 1997] and GOCE [Battrick, 1999] operate in presumably 2002 and 2004, respectively. Nevertheless, a priori error estimates of these models are available to us [Balmino et al., 1998]. From now on we will call these error estimates by the names of their corresponding satellite missions.

The error estimates for GRACE and GOCE are available to degrees 150 and 300, respectively. Because GRACE is expected to have the smallest errors below degree 70 and rapidly increasing errors above, we chose an averaging radius of $r \approx 286 \mathrm{~km}$ as for the EGM96 errors. The GOCE mission is designed to resolve a spatial scale of approximately $100 \mathrm{~km}$, which corresponds to a degree of $l=200$. Consequently, this was our choice for the averaging radius.

The mean sea-surface CLS_SHOM98.2 [Hernandez and Schaeffer, 2000] provides an error estimate $C_{h}$ for the altimetric measurements that is of the order of $2 \mathrm{~cm}$ over the mid-ocean basin and increasing to approxi-

\footnotetext{
${ }^{2}$ http://cddisa.gsfc.nasa.gov/926/egm96/egm96.html
}

mately $4 \mathrm{~cm}$ near the coasts. This estimate was used in all experiments. (Because of the interpolation technique used by Hernandez and Schaeffer, the formal errors can increase to values far above $4 \mathrm{~cm}$ near boundaries where only few data are available. We set those high values to the maximum error of $4 \mathrm{~cm}$. This choice avoids singular behavior of the covariance matrices during filtering and inversion.)

Fig. 6 shows the error covariance matrices for seasurface height - the sum of geoid error and altimetry error, smoothed with the Gaussian filter - -for the three geoid error estimates EGM96, GRACE, and GOCE. For GRACE the variance is almost two orders of magnitude smaller than for EGM96, because the geoid error is almost negligible. In addition to that the higher resolution (smaller averaging radius) of GOCE is visible in the narrow main diagonal of the error covariance matrix. Again, this diagonal consists mainly of errors in $h$ because the GOCE geoid model errors are very small.

\subsection{Minimization}

After the careful definition of $\mathcal{J}$ the minimization routine M1QN3 of the MODULOPT library [Gilbert and Lemaréchal, 1989] was applied. The minimizer searches for the optimal state in the space of independent variables or parameters $p=\left\{T, S, \mathbf{u}_{r e f}, q_{-H}\right\}$, where $T$ is temperature, $S$ salinity, $\mathbf{u}_{r e f}$ are the reference velocities, and $q_{-H}$ is the correction to the boundary condition for the vertical velocity at the sea floor. 
a) EGM96(L=70)

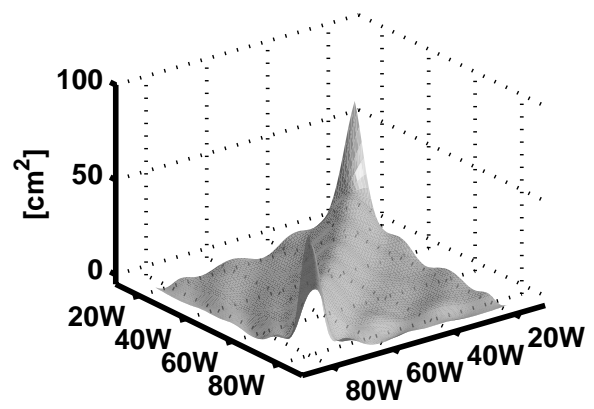

b) $\operatorname{GRACE}(\mathrm{L}=70)$

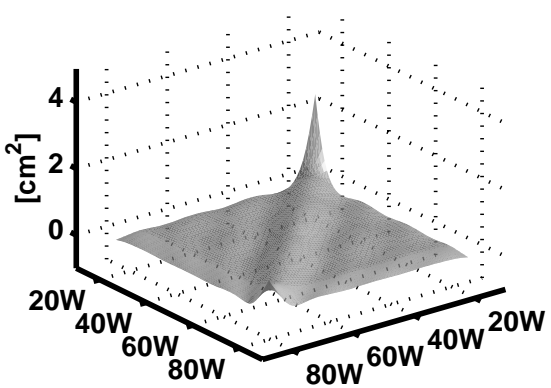

c) $\operatorname{GOCE}(\mathrm{L}=200)$

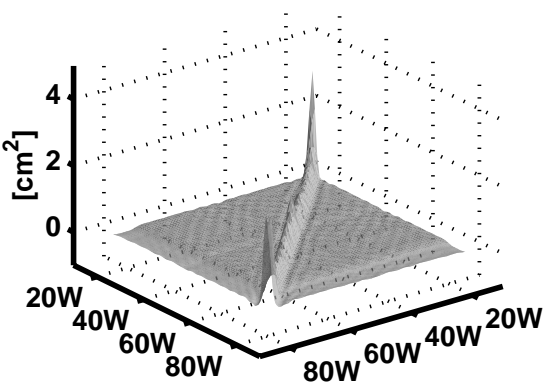

Figure 6. Error covariance matrices for sea-surface height data after filtering of all small scales according to the averaging radius used for a) EGM96, b) GRACE, and c) GOCE.

\subsection{Posterior variances}

Variances of all integral quantities shown in Fig. 7, Section 4 were computed through linear transformations of the error covariance matrix $\mathbf{C}$ between the control variables. $\mathbf{C}$ is identified as the inverse of the Hessian matrix $\mathbf{H}$ associated with the assimilation scheme [Thacker, 1989]. Any observable $\phi$ can be approximated by an expansion around the optimal state of the control parameters $p$ :

$$
\phi \approx \frac{\delta \phi}{\delta p} p+\phi_{0}=\hat{L} p+\phi_{0}
$$

where $\hat{L}$ is a linear operator (in case of a scalar observable, it is a covector). This operator describes the sensitivity of $\phi$ to variations in $p$. Its numerical code can be obtained with the TAMC [Giering, 1999].

Following the standard approach [Thacker, 1989], we assume that the posterior statistics, as well as the prior statistics, are Gaussian and that fluctuations around the optimal state are small in the sense that the original dynamics are well approximated by the linearized equations. Then the error covariance of $\phi$ can be calculated as

$$
\mathbf{C}_{\phi}=\hat{L}^{T} \mathbf{H}^{-1} \hat{L} .
$$

For scalar observables $C_{\phi}$ reduces to a single value: the error variance. As the number of control variables is fairly large (of order $10^{4}$ ), it is computationally prohibitive to calculate $\mathbf{H}$ and especially $\mathbf{H}^{-1}$. However, it is possible to calculate the product of $\mathbf{H}$ with any vector [Le Dimet and Talagrand, 1986; Giering, 1999]. It enables us to use a polynomial approximation of the property $\psi=\mathbf{H}^{-1 / 2} \hat{L}$ from which $C_{\phi}=\psi^{T} \psi$ can be obtained. This procedure is described in detail by Yaremchuk and Schröter [1998].

\section{Experiments}

We now describe a set of four experiments with the non-linear section model. In all experiments the inverse model has to reproduce an annual mean state of the FLAME-model ocean from a snapshot "measurement" of the hydrographic parameters temperature and salinity taken under winter conditions, a three-by-three array of estimates of the mean velocity in the Florida Strait, and an estimate of the reference velocity and the temperature and salinity gradient across the section. Wind fields to calculate Ekman velocities with an assumed Ekman depth of approximately $40 \mathrm{~m}$ are taken from Trenberth et al. [1990]; the bottom slope is calculated by simple central differences from the FLAME model bathymetry.

The first experiment in which no further information is used is called NOSSH. In the other three experiments a one-year mean of sea-surface height data, also taken from the FLAME-model ocean, supplements the model as an additional data constraint on the surface geostrophic velocities. These data are weighted according to the error estimates of the three different geoid models described in the previous section. We call these experiments by the names of the corresponding geoid models: EGM96, GRACE, and GOCE. Because the sea-surface height data are the same for all of the latter three experiments, its effect on the solution will be only due to the different error estimates of Fig. 6 . When evaluating the results, we will focus on mass and temperature flux through the five regions defined in Section 2. Mass and temperature flux values are summarized in Fig. 7. 

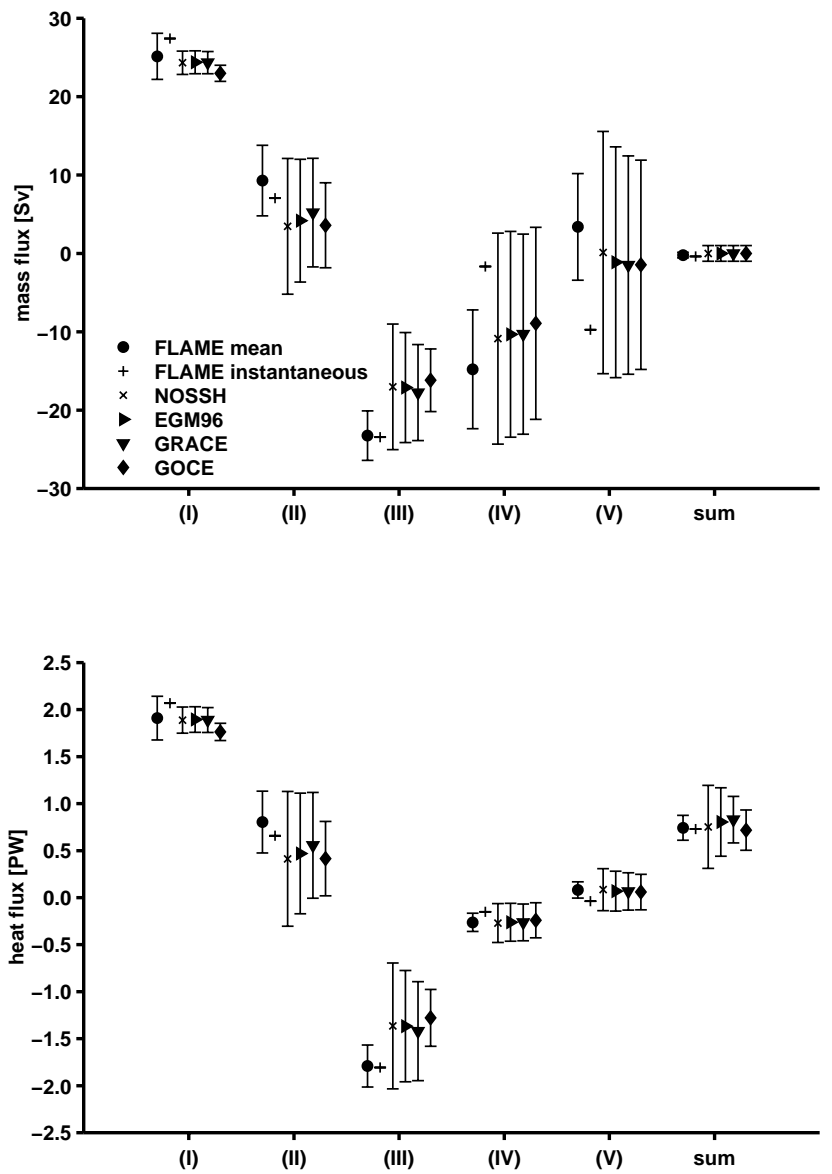

Figure 7. Mass and temperature flux through region I to V: FLAME-model mean with standard deviation, instantaneous values of January 3 , and estimates of the inverse model with error bars.

\subsection{Comparison with the One-Year Mean State of the FLAME Model}

The estimates of mass and temperature flux of all four experiments are generally in agreement with each other within the prior and posterior error bars. In the Florida Strait (region I), the a posteriori estimated errors are small because here the mean velocity field is assumed to be well known from long-term measurements (a priori error of $1 \mathrm{~cm} / \mathrm{s}$ ). The mass flux errors correspond to the estimates of climatological variability of the real Florida Current of $1 \mathrm{~Sv}$ by Schott et al. [1988]. The error of the net mass flux is only $1 \mathrm{~Sv}$ because this quantity has also been assumed well known a priori. In the regions II- $\mathrm{V}$, the estimated mass flux errors can become large, up to $15 \mathrm{~Sv}$ in regions $\mathrm{V}$. The large errors of the individual regions can add up to the small to- tal error of $1 \mathrm{~Sv}$ because of significant anti-correlations (see Section 4.3). In contrast to the mass flux errors, the temperature flux errors in the deep regions IV and V are small. This is a consequence of the specific temperature scale used, namely the Celsius scale, which assigns values near $0^{\circ} \mathrm{C}$ to the deep-sea regions.

For the experiments NOSSH, EGM96, and GRACE, the estimated mass and temperature flux values are different from the FLAME reference values by less than their posterior error estimates. However, the inverse model generally underestimates the mean values. These systematic deviations can have various reasons. The most important one appears to be the fact that small horizontal gradients of the measured density field lead a priori to weak geostrophic shear and thus to weak geostrophic mass flux. These weak velocities cannot become stronger through an inversion that includes penalty terms for roughness. On the contrary: The least-square method itself implicitly leads to smooth solutions [Thacker, 1988; Bennett, 1992, page 301]. The underestimation of the Antilles Current (region II) and the deep western boundary current in region IV can especially be explained this way. Both currents are extremely variable in the FLAME model and have smaller than mean mass fluxes at the time of the "measurement". More information about the mean vertical stratification of the water column, taken for example from a climatological atlas, will be necessary to improve the estimate of the mean circulation. The barotropic information of the sea-surface height data alone appears not to be sufficient.

Different forcing wind fields that lead to different Ekman transports are another cause for the deviations of the inverse model's solutions from the FLAME reference values. The Ekman transport through region III calculated from the climatological wind field of Trenberth et al. [1990] is $6.3 \mathrm{~Sv}$, thus much higher than the 3.9 Sv that one obtains when using the mean of the monthly mean fields that drive the FLAME model. The bigger Ekman transport reduces the overall southward flow through region III.

In experiment GOCE the sea-surface height is available with very high accuracy and resolution. With this data, the formal flux errors are greatly reduced by up to $55 \%$ compared to NOSSH [see also Schröter et al., 2001]. On the other hand, the model's transport estimates in the layers above $1000 \mathrm{~m}$ deviate from the FLAME values by more than the posterior error estimates.

Two a priori assumptions are responsible for these deviations. The a priori error of the height over the 
reference ellipsoid $h$ was estimated to be no larger than $4 \mathrm{~cm}$. Increasing this error near the coastal boundaries could lead to larger posterior flux errors - especially in the Florida Strait - so that here the estimate would become consistent with its errors. Second, the sea-surface height had to be assumed as homogeneous perpendicular to the orientation of the section because the inverse model cannot provide an estimate of the slope of the sea-surface height in that direction. This assumption appears to be too crude in the context of the high resolution of the GOCE geoid model.

The deviations of the flux estimates of the inverse model from the reference values of the FLAME model show that close to the surface some of the model's assumptions are not consistent with accurate sea-surface height data. In addition to those mentioned above, assuming a steady state and the lack of a mixed layer model in the dynamics of the inverse model might cause these deviations. The assumption of thermal wind being the only dynamically relevant process above $1000 \mathrm{~m}$ water depth also appears as too restrictive when using the high precision sea-surface height data in the GOCE experiment. Finally, the model does not take into account turbulent diffusion explicitly in the conservation equations for potential temperature and salinity.

\subsection{Error Reduction due to Additional Sea-Surface Height Data}

Fig. 8 shows the reduction of flux errors that are obtained by using the sea-surface height data with different a priori error estimates in experiments EGM96, GRACE, and GOCE. The reduction is given in percent relative to experiment NOSSH. Schröter et al. [2001] discuss only total fluxes and fluxes through part of the Florida Strait (approximately region I), whereas here this discussion is extended to two vertical layers and five separate regions. A further refinement does not seem reasonable at this point as the inverse model due to its nature is not able to reproduce very small scale properties.

As expected, with increasing accuracy and resolution of the sea-surface height data, the inverse model estimates smaller posterior flux errors. The reduction is greatest in the top layer near the surface (regions I to III), for the absolute velocity at the surface can be determined best with the additional data. Going to greater depths, the errors of the density field (actually the formal errors of temperature and salinity) sum up continuously so that deep velocities and thus fluxes cannot be estimated as accurately as those near the surface. A similar result was found by [LeGrand, 2001].
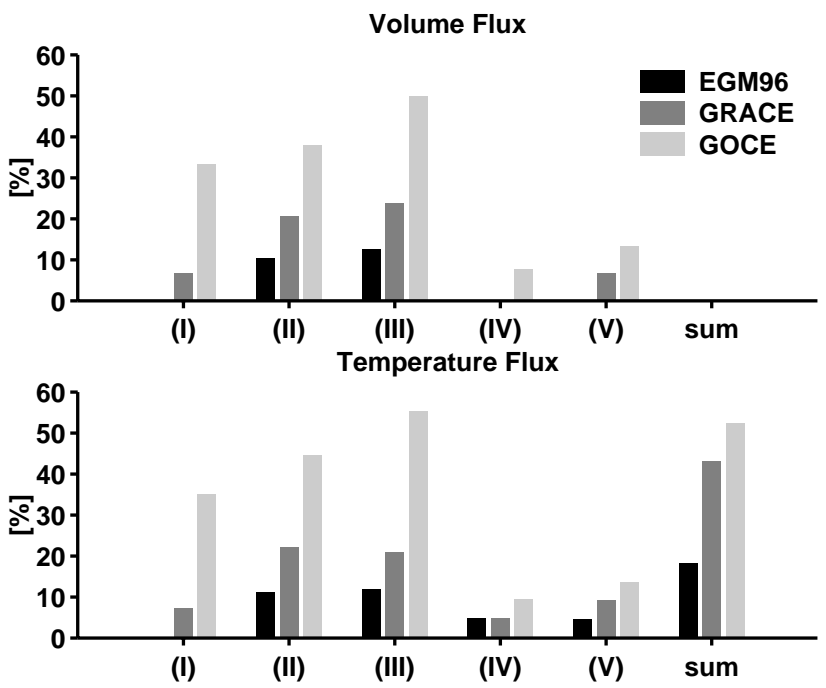

Figure 8. Reduction (in \%) of estimated flux errors relative to NOSSH that are obtained by including seasurface height data with three different error estimates.

The reduction of flux errors relative to experiment NOSSH is least in experiment EGM96. With a more accurate geoid model, represented by the smaller errors of the potential GRACE model, fluxes near the surface can be improved by up to $23 \%$. The total temperature flux, being a truly large scale feature of the section, is more accurate by even $44 \%$. Therefore, with a geoid model as accurate on large scales as the GRACE model, properties with long wavelengths can be estimated with a higher precision than those with small wavelengths. The mass and temperature carried by the Florida Current is assumed well known through current measurements a priori. However, with a high precision, high resolution geoid model such as GOCE, the inverse model can reduce the flux errors through Florida Strait even further by 30-34\%. In regions II and III, the reduction is largest with GOCE, too (up to $55 \%$ ). On the other hand, the effect of the sea-surface height data on the large errors of the fluxes beneath $1000 \mathrm{~m}$ is smaller than $15 \%$.

The total mass flux error of $1 \mathrm{~Sv}$ prior to the inversion cannot be reduced, not even with the "best" data in experiment GOCE. The total temperature flux, however, for which no a priori assumption has been used, can be improved with sea-surface height data by up to $51 \%$ with GOCE over experiment NOSSH without seasurface height data. With the less accurate EGM96 this improvement still is $18 \%$. 
FLAME
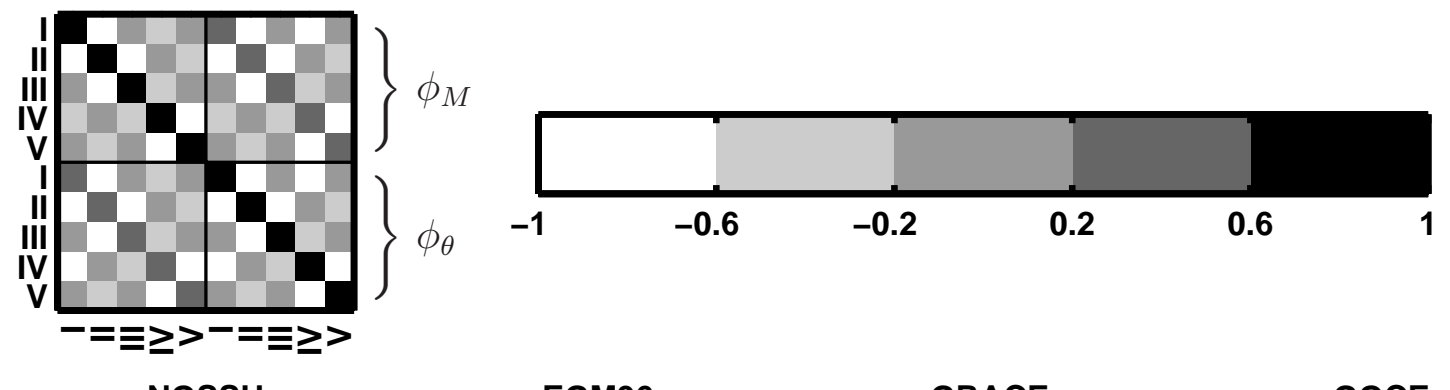

NOSSH

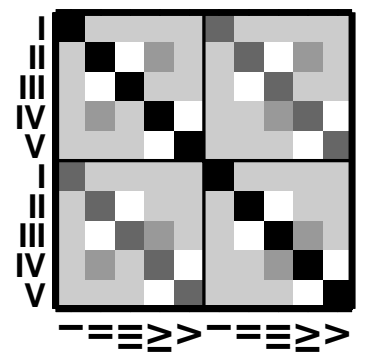

EGM96

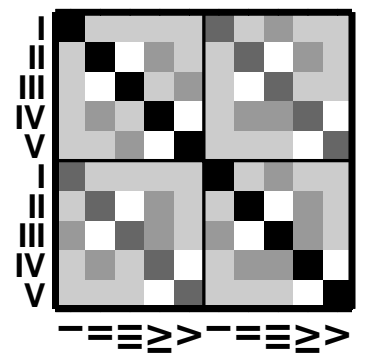

GRACE

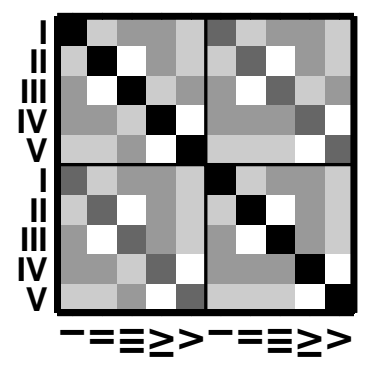

GOCE

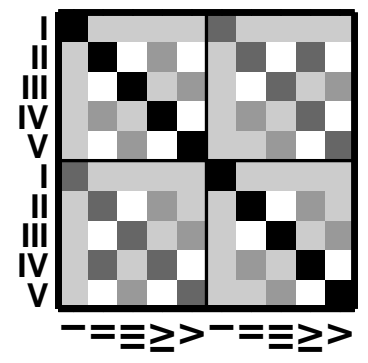

Figure 9. Correlations of the flux errors: the first five columns and rows describe the correlations of the five mass fluxes $\phi_{M}$ through regions I - V; the last five columns and rows, the corresponding temperature fluxes $\phi_{\theta}$.

\subsection{Posterior Error Correlations}

4.3.1. FLAME reference Fig. 9 shows the posterior error covariances of the mass and temperature fluxes normalized by their variances. These matrices describe the correlation $C_{m n} / \sqrt{C_{m m} C_{n n}}$ between the $m$-th and $n$-th flux error. Also shown as a reference are the correlations of the standard deviations of the FLAME model from its one-year mean.

In the FLAME reference case, all mass fluxes are anti-correlated to the fluxes through their respective horizontal neighboring region. The vertical correlation on the other hand is always positive; however, it is not greater than 0.6. This vertical correlation is interpreted as a manifestation of the barotropic variability of the current field which dominates the overall vertical fluctuations of the flow. The fluctuations of the fluxes above $1000 \mathrm{~m}$ are out of phase for neighboring regions: in the statistical mean, a strong Florida Current comes along with a weak Antilles' Current and strong recirculation in the eastern Atlantic. The correlation of the Florida Current with the flows through the deep regions IV and $\mathrm{V}$ is a consequence of their vertical coupling with the fluxes through respective regions above $1000 \mathrm{~m}$. The strong horizontal anti-correlation also shows up in the eigenvalue decomposition of the error covariance matrices (Fig. 10, top panel). Here the eigenvectors of the first two eigenvalues, which together describe $94 \%$ of the variance, display the negative correlation clearly.

4.3.2. Solutions of the inverse model Up to now, the variances of the fluxes in the solution of the inverse model have been conceived to be formal errors. Now they shall be interpreted as the deviation from the estimated mean value due to variability. This allows us to treat the statistical covariances of the inverse model as temporal properties of the estimated fluxes.

In the experiment NOSSH without sea-surface height data, some of the correlations of the FLAME-model cannot be completely reproduced. The horizontal anticorrelation between regions II and III or IV and V are present both in the FLAME-model and in the inverse model, as can also be seen in the eigenvalue decomposition of the mass flux error covariance matrices: The eigenvectors of the two largest eigenvalues - describing together almost $94 \%$ of the total variance - are similar for the FLAME and the NOSSH panel in Fig. 10. However, there is almost no correlation, represented by the small forth eigenvalue in the NOSSH panel in Fig. 10, between the Florida Strait and the remaining regions of the section in the inverse model solution. This is due to 

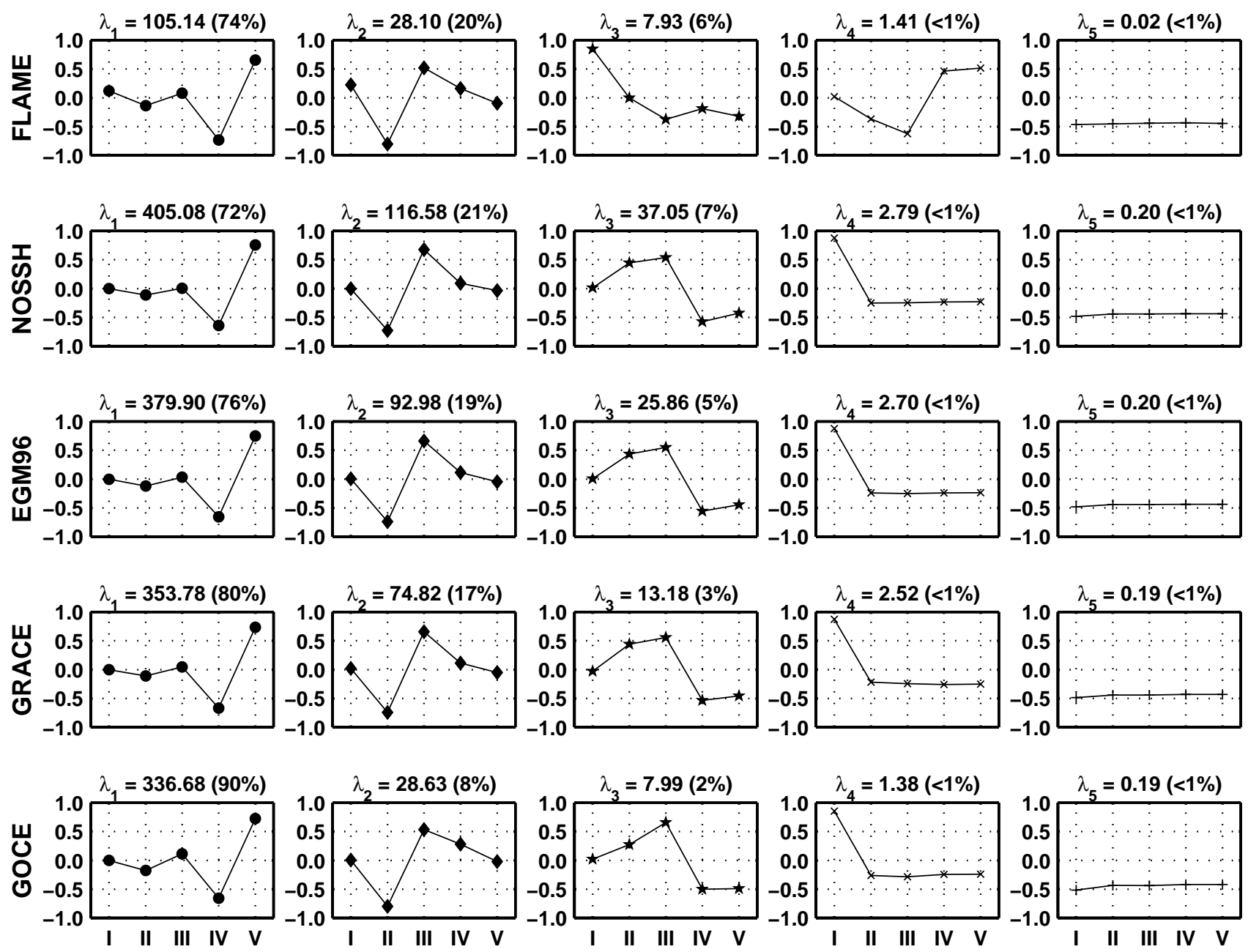

Figure 10. Eigenvalue decomposition of the mass flux error covariance matrices: for the FLAME reference case and for each experiment, there are five plots of the eigenvectors in one row. The size of the eigenvalues (in $\left[10^{6} \mathrm{~m}^{3} / \mathrm{s}\right]^{2}$ ) and the percentage of the variance they describe is given above each eigenvector plot.

the fact that the velocity field and the fluxes are determined independently by the current measurement. Vertical correlations of the inverse model are weaker than in the FLAME-model. For regions III and $\mathrm{V}$ the correlation is even negative (not resolved by the gray scale in Fig. 9). This negative correlation stems from fluctuations of the vertical shear of the geostrophic velocities due to allowed variability (non-zero a priori error) of the hydrographic fields.

With the additional sea-surface height data the inverse model can improve the estimates of some of the correlations of the FLAME-model. In particular, the new data constrain the barotropic component of the flow so that the positive vertical correlations are re- tained.

In Section 4.1, a systematic underestimation of fluxes outside of Florida Strait was described. By analyzing the posterior error covariances we get an idea of how this underestimation comes about. The horizontal anti-correlation between fluxes through horizontally neighboring regions points to a mechanism, by which the inverse model satisfies the constraint of zero net mass flux: the inverse model estimates too small fluxes through regions II and IV. One reason for this was that weak horizontal density gradients lead too weak vertical shear of geostrophic velocities that in turn lead to too small fluxes. These small fluxes are compensated by fluxes east of $73^{\circ} \mathrm{W}$, which consequently must also 


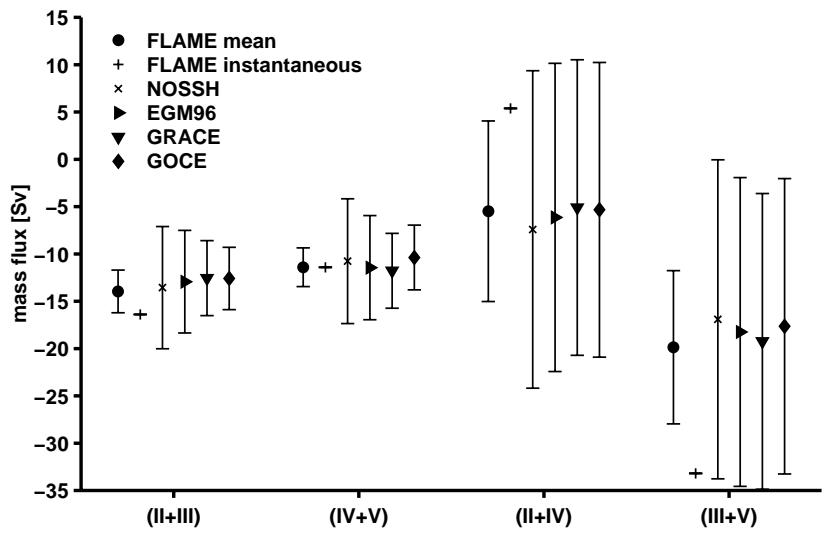

Figure 11. Mass flux through combinations of regions I to V: FLAME-model mean with standard deviation, instantaneous values of January 3, and estimates of the inverse model with error bars.

be too small in order to get the correct net mass flux. By the horizontal anti-correlation we can state that a larger flux to the north west of $73^{\circ} \mathrm{W}$ will automatically lead to a larger southward flux east of $73^{\circ} \mathrm{W}$ and vice versa. This anti-correlation is even increased when using GOCE data, which explains that despite this accurate data this part of the solution cannot be improved (see Fig. 10, bottom panel). Because of the horizontal anti-correlation, the estimates of the horizontal sum of the fluxes (region II + III and IV + V) agree much more with the FLAME reference values than the estimates of the vertical sum (Fig. 11), and the net mass flux can be estimated correctly to be zero in spite of an estimate of the sub-tropical gyre that is too weak.

\section{Summary and Conclusion}

An inverse model was described that estimates a mean ocean state along a hydrographic section. The choice of independent parameters allows this model to estimate not only the flow through the section but also new temperature and salinity fields that deviate from measurements as the latter do not represent a mean. Here, these deviations have only been discussed insofar as they influence the flow field and contribute to the overall error of flux error estimates. The fluxes and their errors can be estimated more reliably than from the standard box inverse models, which have only velocities as unknown parameters. More reliable error estimates will help interpret fluxes that are inferred from hydrographic data.

In addition to hydrographic data, the model includes sea-surface height data in its estimates. The corresponding measurement to be assimilated is the difference between the measured sea-surface height over a reference ellipsoid and a marine geoid. The presently available geoid model EGM96 has large uncertainties associated with it. On the other hand, there is the prospect of new geoid models for which there are error estimates available, but no data yet. As a consequence we have chosen a synthetic data set produced by an ocean general circulation model to investigate the ability of the inverse model to reproduce a reference mean state along a transatlantic hydrographic section at $24^{\circ} \mathrm{N}$. In the inverse scheme, the sea-surface height data were weighted by different error estimates corresponding to errors of geoid models that differ in accuracy and resolution: EGM96, and that of the future GRACE and GOCE missions.

The estimates of fluxes through five regions of the section by the inverse model are mostly consistent with the reference state within their large posterior error estimates. With sea-surface height data the posterior errors reduce. However, with the most accurate data that will be available with GOCE the errors become smaller than the deviation of the inverse model's estimate from the reference. In this case, the model assumptions need to be reviewed critically. Geostrophy and the model's stationarity appear as approximations, that are too crude in the context of the very precise sea-surface height data available with GOCE. Furthermore the assumption of horizontal homogeneity of the sea-surface height data normal to section seems to be too strong for the very short scales resolved by GOCE.

The analysis of the posterior error covariance estimates of the inverse model provides further information on the estimated flow field. Interpreting the posterior error estimates of the inverse model as temporal variances and comparing them to the FLAME model's covariances also gives substantial insight into some of the mechanisms of the inverse model and provide explanations for some of the inverse model's drawbacks. Because of the constraint on the net mass flux the underestimation of a flux in one region is compensated horizontally by underestimating a flux going the opposite direction through another region. In this way, the inverse model underestimates the strength of the subtropical gyre and cannot reproduce the vertical correlations of fluxes correctly. The flux through Florida Strait in the inverse model is almost completely determined by velocity measurements and thus, in contrast to the FLAME model, decoupled from the remainder of the section. 
We have shown in this paper that the non-linear inverse section model can yield consistent estimates of a mean state from synoptic hydrographic measurements and a mean sea-surface height derived from a time series. However, if the sea-surface height data are too precise, the assumptions of the stationary, geostrophic inverse model appear to be too crude. Only taking into account all model errors associated with these assumptions will lead to consistent results in the case of very precise data. On the other hand, with additional a priori errors the posterior errors might increase as well. From this point of view, the estimated errors of the fluxes in experiments NOSSH, EGM96, and GRACE can be interpreted as a lower error bound that one can achieve when estimating fluxes with a stationary, geostrophic model from synoptic hydrographic measurements. In order to improve estimates of the state of the ocean over this bound - as it will be possible with the geoid model GOCE - more complex inverse models of the ocean circulation will be necessary.

\section{Appendix: Model Equations and Cost Function}

\section{A1. Equations}

The inverse model calculates density from the nonlinear equation of state of sea-water [Fofonoff and Millard, 1983] as a function of salinity $S$, in situ temperature $T$, and pressure $p$ (depth). According to the thermal wind equation, the vertical shear of horizontal velocity is proportional to the horizontal density gradient. Integrating the thermal wind equation yields

$$
\mathbf{u}_{g}=\mathbf{u}_{r e f}+\frac{g}{\rho_{0} f} \int_{-H}^{z} \mathbf{k} \times \nabla \rho d z .
$$

with the unknown reference velocities $\mathbf{u}_{r e f} . \quad f=$ $2 \Omega \sin \phi$ is the Coriolis parameter that depends on latitude $\phi . g$ is the acceleration resulting from gravity and $\rho_{0}$ a mean density, in accordance with the Boussinesq approximation [see e.g., Gill, 1982]. The local coordinate system is oriented along the section, so that the $x$ direction is parallel to the section (unit vector $\mathbf{i}$ ) and the $y$ direction normal to it (unit vector $\mathbf{j}$ ). The vertical unit vector is $\mathbf{k}$. The horizontal geostrophic current vector can be written as $\mathbf{u}_{g}=u_{g} \mathbf{i}+v_{g} \mathbf{j}(+0 \mathbf{k})$.

The "geostrophic" vertical velocity $w_{g}$ can be calculated from integration of the planetary vorticity equation

$$
f \frac{\partial w_{g}}{\partial z}-\nabla_{h} f \cdot \mathbf{u}_{g}=0 .
$$

At this point we take a different approach than Nechaev and Yaremchuk [1995] and do not differentiate this equation to get a new equation with two boundary conditions. Instead, we integrate (A2) from the bottom $(z=-H)$ with boundary condition

$$
w_{g}(-H)+\left(\mathbf{u}_{r e f} \cdot \nabla_{h} H+q_{-H}\right)=0
$$

where unknown errors $q_{-H}$ are allowed. These stem from inaccurate estimates of the bottom slope $\nabla H$ and from neglecting Ekman pumping. The solution

$$
w_{g}(z)=w_{g}(-H)+\frac{\nabla f}{f} \int_{-H}^{z} \mathbf{u}_{g}\left(z^{\prime}\right) d z^{\prime}
$$

satifies the surface boundary condition at $z=0$ only approximately. According to Pedlosky [1996] the geostrophic vertical component $w_{g}(0)$ and the winddriven Ekman component $w_{e k}(0)=-(\mathbf{k} \times \nabla)(\boldsymbol{\tau} / f) / \rho_{0}$ cancel each other at the surface, so that the surface boundary condition reads

$$
w_{g}(0)-\left(\frac{(\mathbf{k} \times \nabla)(\boldsymbol{\tau} / f)}{\rho_{0}}+q_{\boldsymbol{\tau}}\right)=0 .
$$

The error $q_{\tau}$, which can be interpreted as wind stress error, is subject to minimization.

Potential temperature $\theta=\theta\left(S, T, p, p_{0}\right)$ relative atmospheric pressure $p_{0}$ and salinity $S$ are conservative properties [see e.g. Gill, 1982]. In this model, they have to obey the stationary advective balance

$$
\mathbf{u} \cdot \nabla_{h} C_{n}+w \frac{\partial C_{n}}{\partial z}=q_{n}
$$

with $\mathbf{u}=\mathbf{u}_{g}+\mathbf{u}_{e k}$ and $w=w_{g}+w_{e k}$. The unknown source terms $q_{n}$ describe processes for the hydrographic parameters $C_{n}=\theta, S$ that the model cannot resolve. These are for example turbulent diffusion and chemical and biological processes. $\mathbf{u}_{e k}$ is the directly wind-driven near-surface velocity of the Ekman spiral [see e.g. Pedlosky, 1987, pp. 226]. The vertical Ekman velocity $w_{e k}$ follows from the equation of continuity [Pedlosky, 1996]:

$$
\begin{aligned}
\frac{\partial w_{e k}}{\partial z} & =-\nabla \cdot \mathbf{u}_{e k} \\
\text { with } w_{e k}(0) & =-\frac{(\mathbf{k} \times \nabla)(\boldsymbol{\tau} / f)}{\rho_{0}} .
\end{aligned}
$$

The slow, large scale motion calculated by the inverse model has to meet another constraint: a prior estimate of total mass transport $\phi_{V}$ normal to the section

$$
\iint_{\Omega} v d z d x=\left(\phi_{V}+\delta \phi_{V}\right) .
$$


Here $\delta \phi_{V}$ is an unknown error of this estimate, $\Omega$ the area of the section. Temperature flux (or rather internal energy flux) of a section of vanishing net mass transport is estimated as the product of velocity, density, specific heat at constant pressure $c_{p}$, and potential temperature [Warren, 1999]:

$$
\phi_{\theta}=\iint_{\Omega} v \rho c_{p} \theta d z d x .
$$

The geostrophic velocity $v_{g}$ at the surface is directly proportional to the slope of the sea-surface $\zeta$ :

$$
v_{g}=\frac{g}{f} \frac{\partial \zeta}{\partial x} .
$$

In this paper, the model variable $\zeta$ is determined by integration of this geostrophic equation:

$$
\zeta(x)-\hat{P} \mathbf{u}=0
$$

with

$$
\begin{aligned}
\hat{P} \mathbf{u}= & \int_{0}^{x} \frac{f}{g} v_{g}\left(x^{\prime}, z=0\right) d x^{\prime} \\
& -\frac{1}{L} \int_{0}^{L} \int_{0}^{x^{\prime}} \frac{f}{g} v_{g}\left(x^{\prime \prime}, z=0\right) d x^{\prime \prime} d x^{\prime},
\end{aligned}
$$

where $L$ is the total length of the section. This way, data and model values are normalized so that they have zero mean. The wind-driven Ekman velocity at the surface does not generate a pressure boundary layer and sea-surface elevation [Wunsch and Stammer, 1998].

\section{A2. Cost Function and Adjoint Method}

The individual terms of cost function (1) are in detail:

a) data terms (the $\hat{\Phi}$ 's map the model variables to the data points)

$$
\begin{gathered}
\mathcal{J}_{C}=\frac{1}{2} \sum_{m, n} \int_{0}^{L} \int_{-H}^{0} \int_{-H}^{0} d z d z^{\prime} d x \ldots \\
\ldots\left(\hat{\Phi}_{C} C_{m}(x, z)-C_{m}^{*}(x, z)\right) \ldots \\
\ldots W_{m n}\left(x, z, z^{\prime}\right)\left(\hat{\Phi}_{C} C_{n}\left(x, z^{\prime}\right)-C_{n}^{*}\left(x, z^{\prime}\right)\right) \\
\mathcal{J}_{\zeta}=\frac{1}{2} \int_{0}^{L} \int_{0}^{L}\left(\hat{\Phi}_{\zeta} \zeta(x)-\zeta^{*}(x)\right) \ldots \\
\ldots . W_{\zeta}\left(x, x^{\prime}\right)\left(\hat{\Phi}_{\zeta} \zeta\left(x^{\prime}\right)-\zeta^{*}\left(x^{\prime}\right)\right) d x^{\prime} d x \\
\mathcal{J}_{\mathbf{u}}=\frac{1}{2} \int_{\Omega}\left(\hat{\Phi}_{\mathbf{u}} \mathbf{u}-\mathbf{u}^{*}\right) W_{\mathbf{u}}\left(\hat{\Phi}_{\mathbf{u}} \mathbf{u}-\mathbf{u}^{*}\right) d x d z
\end{gathered}
$$

b) "soft" constraints

$$
\begin{aligned}
\mathcal{J}_{\boldsymbol{\tau}} & =\frac{1}{2} \int_{0}^{L} W_{\boldsymbol{\tau}} q_{\boldsymbol{\tau}}^{2} d x \\
\mathcal{J}_{-H} & =\frac{1}{2} \int_{0}^{L} W_{-H} q_{-H}^{2} d x \\
\mathcal{J}_{q} & =\frac{1}{2} \sum_{n} \iint_{\Omega} W_{q_{n}} q_{n}^{2} d z d x \\
\mathcal{J}_{\phi_{V}} & =\frac{1}{2} W_{\delta \phi_{V}}\left(\delta \phi_{V}\right)^{2}
\end{aligned}
$$

c) regularization

$$
\begin{aligned}
\mathcal{J}_{\mathbf{u}_{\text {ref }}} & =\frac{1}{2} \int_{0}^{L} W_{\mathbf{u}_{\text {ref }}}\left(\mathbf{u}_{r e f}-\mathbf{u}_{r e f}^{*}\right)^{2} d x \\
\mathcal{J}_{\partial_{y} C} & =\frac{1}{2} \sum_{n} \iint_{\Omega} W_{\partial_{y} C_{n}}\left(\frac{\partial C_{n}}{\partial y}-\frac{\partial C_{n}}{\partial y}\right)^{2} d z d x \\
\mathcal{J}_{\hat{S} C} & =\frac{1}{2} \sum_{n} \iint_{\Omega} W_{\hat{S} C_{n}}\left(\hat{S}_{c} C_{n}\right)^{2} d z d x \\
\mathcal{J}_{\hat{S} q} & =\frac{1}{2} \sum_{n} \iint_{\Omega} W_{\hat{S} q_{n}}\left(\hat{S}_{q} q_{n}\right)^{2} d z d x \\
\mathcal{J}_{\hat{S} \mathbf{u}} & =\frac{1}{2} \iint_{\Omega} W_{\hat{S} \mathbf{u}}\left(\hat{S}_{u} \mathbf{u}\right)^{2} d z d x
\end{aligned}
$$

Here, $C_{n}$ stands for temperature and salinity, $W_{m n}$ for their nondiagonal weights. $q_{-H}, q_{\boldsymbol{\tau}}$, and $q_{n}$ are the residuals of equations (A2) to (A4) and $\hat{S}_{\psi}$ are the differential smoothness operators that act on the tracer fields, the tracer residuals, and the velocity field. $\iint_{\Omega} d x d z$ denotes integration over the section plane $\Omega$. Summations are performed over the hydrographic parameters temperature and salinity, which are labeled by $n$ (or $m)$.

The model equations (A1) through (A9) can be summarized into an operator $\hat{M}$ that maps the independent 
model parameter vector $\mathbf{p}$ onto the data vector $\mathbf{d}$. The cost function can be symbolically written as

$$
\mathcal{J}=\frac{1}{2}(\hat{M}(\mathbf{p})-\mathbf{d})^{T} \mathbf{W}(\hat{M}(\mathbf{p})-\mathbf{d}) .
$$

Variation of the cost function yields the gradient of the cost function with respect to $\mathbf{p}$ :

$$
\nabla \mathcal{J}=\left(\frac{\partial \hat{M}}{\partial \mathbf{p}}\right)^{T} \mathbf{W}(\hat{M}(\mathbf{p})-\mathbf{d}) .
$$

In our case, the numerical code of the Jacobi- or adjoint operator $(\partial \hat{M} / \partial \mathbf{p})^{T}$ is generated automatically by the Tangent-linear Adjoint Model Compiler [TAMC, Giering, 1999]. An iterative algorithm searches for the minimum of $\mathcal{J}$, where $\nabla \mathcal{J}=0$.

\section{Acknowledgments.}

The authors thank Alexey Yaremchuk for his help with computing $\mathbf{H}^{-1 / 2}$, the square root of the inverse of the Hessian matrix. We acknowledge the valuable contribution of Ralf Giering's Tangent linear Adjoint Compiler [Giering, 1999$]$ in speeding the production of the adjoint code to the model. Thanks are due to Lisa McFarren for correcting many errors in the original manuscript. FLAME model developement and integration was part of the Sonderforschungsbereich 460: Dynamics of Thermohaline Circulation Variability at Kiel University.

\section{References}

Balmino, G., F. Perosanz, R. Rummel, N. Sneeuw, H. Sünkel, and P. Woodworth, European views on dedicated gravity field missions: GRACE and GOCE, An Earth Sciences Division Consultation Document, ESA, ESD-MAG-REP-CON-001, 1998.

Barnier, B., L. Siefridt, and P. Marchesiello, Thermal forcing for a global ocean circulation model using a three-year climatology of ECMWF analyses, Journal of Marine Systems, 6, 363-380, 1995.

Battrick, B. (Ed.), Gravity Field and Steady-State Ocean Circulation Explorer, ESA SP-1233 (1) - The Four Candidate Earth Explorer Core Missions, ESA, c/o ESTEC, Noordwijk, The Netherlands, 1999.

Bennett, A. F., Inverse Methods in Physical Oceanography, Cambridge University Press, 1992.

Bindoff, N. L., and T. J. McDougall, Diagnosing climate change and ocean ventilation using hydrographic data, Journal of Physical Oceanography, 24, 1137-1152, 1994.

Böning, C. W., and R. Budich, Eddy dynamics in a primitive equation model: Sensitivity to horizonal resolution and friction, Journal of Physical Oceanography, 22, 361-381, 1992.

Böning, C. W., C. Dieterich, B. Barnier, and Y. Jia, Seasonal cycle of meridional heat transport in the subtropical
North Atlantic: a model intercomparison in relation to observations near $25^{\circ} \mathrm{N}$, Progress In Oceanography, 48 , 231-253, 2001.

Boyer, T. P., and S. Levitus, Objective analyses of temperature and salinity for the word ocean on a $1 / 4$ degree grid, NOAA Atlas Series NESDIS 11, U.S. Gov. Printing Office, Washington, D.C., 1997.

Bryden, H. L., Ocean heat transport across $24^{\circ} \mathrm{N}$ latitude, in Interactions Between Global Climate Subsystems, edited by G. A. McBean and M. Hantel, vol. 75 of AGU Geophysical Monograph, pp. 65-75, American Geophysical Union, Washington, DC, 1993.

de las Heras, M. M., and R. Schlitzer, On the importance of intermediate water flows for the global ocean overturning, Journal of Geophysical Research, 104, 15,51515,536, 1999.

Fofonoff, P., and J. Millard, R.C., Algorithms for computation of fundamental properties of seawater, Unesco Technical Papers in Marine Science, 53 pp. 44, Unesco, 1983.

Ganachaud, A., and C. Wunsch, Improved estimates of global ocean circulation, heat transport and mixing from hydrographic data, Nature, 408, 453-457, 2000.

Ganachaud, A., C. Wunsch, M.-C. Kim, and B. Tapley, Combination of TOPEX/POSEIDON data with a hydrographic inversion for determination of the oceanic general circulation and its relation to geoid accuracy, Geophysical Journal International, 128, 708-722, 1997.

Giering, R., Tangent linear and Adjoint Model Compiler, Users manual 1.4, 1999, http://puddle.mit.edu/ ralf/tamc.

Gilbert, J. C., and C. Lemaréchal, Some numerical experiments with variable-storage quasi-Newton algorithms, Mathematical Programming, 45, 407-435, 1989.

Gill, A. E., Atmosphere-Ocean Dynamics, vol. 30 of International Geophysics Series, Academic Press, 1982.

Hernandez, F., and P. Schaeffer, Altimetric mean sea surfaces and gravity anomaly maps inter-comparisons, AVISO technical report AVI-NT-011-5242-CLS, CLS, Toulouse, France, 2000.

Jekeli, C., Alternative methods to smooth the Earth's gravity field, Tech. Rep. 327, Department of Geodetic Science and Surveying, Ohio State University, 1981.

Le Dimet, F.-X., and O. Talagrand, Variational algorithms for analysis and assimilation of meteorological observations: Theoretical aspects, Tellus, 38A, 97-110, 1986.

LeGrand, P., Impact of the Gravity Field and Steady-State Ocean Circulation Explorer (GOCE) mission on ocean circulation estimates: Volume fluxes in the climatological inverse model of the Atlantic, Journal of Geophysical Research, 106, 19,597-19,610, 2001.

Lemoine, F. G., et al., The development of the NASA GSFC and NIMA joint geopotential model, in Gravity, geoid and marine geodesy, edited by J. Segawa, H. Fujimoto, and S. Okubo, vol. 117, pp. 461-469, International Association of Geodesy Symposia, Springer, New York, 1997.

Macdonald, A. M., The global ocean circulation: a hydrographic estimate and regional analysis, Prog. Oceanogr., 41, 281-382, 1998. 
Macdonald, A. M., and C. Wunsch, An estimate of the global ocean circulation and heat flux, Nature, 382, 436439, 1996.

McIntosh, P. C., and S. R. Rintoul, Do box inverse models work?, Journal of Physical Oceanography, 27, 291-308, 1997.

Nechaev, D. A., and M. I. Yaremchuk, Application of the adjoint technique to processing of a standard section data set: World Ocean Circulation Experiment section S4 along $67^{\circ} \mathrm{S}$ in the Pacific Ocean, Journal of Geophysical Research, 100, 865-879, 1995.

Pacanowski, R., MOM 2 Documentation (User's guide and reference manual), GFDL Ocean Technical Reports 3, Princeton University, 1995.

Pedlosky, J., Geophysical Fluid Dynamics, Springer, New York, 1987, 710 pp.

Pedlosky, J., Ocean Circulation Theory, Springer, Berlin, 1996, 453 pp.

Redler, R., K. Ketelsen, J. Dengg, and C. Böning, A high-resolution numerical model for the circulation of the Atlantic Ocean, in Proceedings in the Fourth European $S G I / C R A Y$ MPP Workshop, edited by H. Lederer and F. Hertweck, pp. 95-108, Max-Planck-Institut für Plasmaphysik, Garching, Germany, 1998, http://www.rzg.mpg.de/mpp-workshop/papers/ippreport.html.

Rintoul, S. R., South Atlantic interbasin exchange, J. Geophys. Res., 96, 2675-2592, 1991.

Roemmich, D., and C. Wunsch, Two transatlantic sections: Meridional circulation and heat flux in the subtropical North Atlantic Ocean, Deep-Sea Research, 32, 619-664, 1985 .

Schott, F. A., T. N. Lee, and R. Zantopp, Variability of structure and transport of the Florida Current in the period range of days to seasonal, Journal of Physical Oceanography, 18, 1209-1230, 1988.

Schröter, J., M. Losch, and B. M. Sloyan, Impact of the GOCE gravity mission on ocean circulation estimates: Part II. Volume and heat fluxes across hydrographic sections of unequally spaced stations, accepted by Journal of Geophysical Research, 2001.

Sloyan, B. M., and S. R. Rintoul, The Southern Ocean limb of the global deep overturning circulation, Journal of Physical Oceanography, 31, 143-173, 2001.

Tapley, B. D., The gravity recovery and climate experiment (GRACE), Supplement to EOS Transactions of the American Geophysical Union, 78, 163, 1997.

Thacker, W. C., Fitting models to inadequate data by enforcing spatial and temporal smoothness, Journal of Geophysical Research, 93, 10,556-10,566, 1988.
Thacker, W. C., On the role of the Hessian matrix in fitting models to data, Journal of Geophysical Research, 94, 6177-6196, 1989.

Trenberth, J. M., J. Olson, and W. G. Large, The mean annual cycle in Global Ocean wind stress, Journal of Physical Oceanography, 20, 1742-1760, 1990.

Wahr, J., M. Molenaar, and F. Bryan, Time variability of the Earth's gravity field: Hydrological and oceanic effects and their possible detection using GRACE, Journal of Geophysical Research, 103, 30,205-30,299, 1998.

Warren, B. A., Approximating the energy transfer across oceanic sections, Journal of Geophysical Research, 104, 7915-7919, 1999.

Webb, D. J., et al., Using an eddy resolving model to study the Southern Ocean, EOS, 72, 169, 174, 175, 1991.

Willebrand, J., B. Barnier, C. Böning, C. Dieterich, P. D. Killworth, C. LeProvost, Y. Jia, J.-M. Molines, and A. L. New, Circulation characteristics in three eddy-permitting models of the North Atlantic, Progress in Oceanography, 48, 123-161, 2001.

Wunsch, C., The North Atlantic general circulation west of $50^{\circ}$ determined by inverse methods, Reviews of Geophysics and Space Physics, 16, 583-620, 1978.

Wunsch, C., and D. Stammer, Satellite altimetry, the marine geoid, and the oceanic general circulation, Annual Reviews of Earth and Planetary Science, 26, 219-253, 1998.

Yaremchuk, A. I., and J. Schröter, Spectral analysis of symmetric operators: Application to the Laplace tidal model, Journal of Computational Physics, 14\%, 1-21, 1998.

Yaremchuk, M., D. Nechaev, J. Schröter, and E. Fahrbach, A dynamically consistent analysis of circulation and transports in the southwestern Wedell Sea, Annales Geophysicae, 16, 1024-1038, 1998.

Yaremchuk, M. I., N. L. Bindoff, J. Schröter, D. Nechaev, and S. R. Rintoul, On the zonal and meridional circulation and ocean transports between Tasmania and Antarctica, Journal of Geophysical Research, 106, 2815-2832, 2001.

Zhang, H.-M., and N. G. Hogg, Deducing dynamic properties form simulated hydrogaphic data: Part I. results from a non-eddy-resolving model, Journal of Marine Research, 54, 679-703, 1996.

This preprint was prepared with AGU's LATEX macros v5.01, with the extension package 'AGU ${ }^{++}$' by P. W. Daly, version $1.6 \mathrm{~b}$ from 1999/08/19. 